\title{
AVALIAÇÃO DE UM MODELO DE PASSO INVARIANTE NA PREDIÇÃO DA ESTRUTURA DE UM POVOAMENTO DE Eucalyptus sp. ${ }^{1}$
}

Thelma Shirlen Soares ${ }^{2}$, Helio Garcia Leite ${ }^{3}$, Antônio Bartolomeu do Vale ${ }^{3}$, Carlos Pedro Boechat Soares ${ }^{3}$ e

Gilson Fernandes da Silva ${ }^{4}$

\begin{abstract}
RESUMO - O objetivo deste estudo foi avaliar o modelo de distribuição diamétrica de passo invariante proposto por Guimarães (1994) na projeção da produção de um povoamento de Eucalyptus sp., simulando as alterações nas estruturas horizontal e vertical ao longo do tempo. Utilizaram-se dados da primeira rotação de povoamentos de eucalipto híbrido estaca (Eucalyptus grandis x Eucalyptus urophylla), plantados no espaçamento 3,0 x 2,0 m, localizados na região noroeste do Estado da Bahia, provenientes de medições anuais com idade de medição variando entre 25 e 89 meses. Para realizar as prognoses, foram empregados percentis tomados a 50 e $75 \%$ da distribuição diamétrica e as alturas correspondentes aos diâmetros nessas posições. Verificou-se que o modelo de projeção é factível e pode ser utilizado com eficiência, já que ocorreram tendências semelhantes entre os volumes prognosticados e os observados nas parcelas. Além disso, devido à sua simplicidade e à compatibilidade dos resultados, recomenda-se a sua utilização na projeção do crescimento e produção de Eucalyptus sp.
\end{abstract}

Palavras-chave: Crescimento e produção, distribuição diamétrica e eucalipto.

\section{EVALUATION OF A STEP INVARIANT MODEL FOR THE PREDICTION OF EUCALYPTUS STAND STRUCTURE}

\begin{abstract}
This study was carried out to evaluate the step-invariant diametric distribution model, proposed by Guimarães (1994), for the prediction of a Eucalyptus stand production, simulating the alterations in the horizontal and vertical structures with time. Data were taken from annual measurements, with measurement age between 25 and 89 months, of the first rotation of hybrid eucalyptus stands (Eucalyptus grandis $x$ Eucalyptus urophylla), plant spacing of $3.0 \times 2.0 \mathrm{~m}$, located in the northeast region of State of Bahia. To perform prognoses percentiles were taken at 50 and $75 \%$ of the diametric distribution, and the heights corresponding to the diameters in these positions. The prediction model was proved to be feasible for efficient use, since there were similar tendencies between the forecasted and the observed volumes in the plots. Besides, its use in growth and production prediction of Eucalyptus sp. studies is recommended for its simplicity and result compatibility.
\end{abstract}

Keywords: Growth and yield, diametric distribution and eucalyptus.

\section{INTRODUÇÃO}

O rendimento sustentável das florestas requer não somente um conhecimento do estoque de crescimento da floresta, mas também um conhecimento das produções esperadas no futuro (ASSMANN, 1970). Assim, a necessidade de planejamento da produção florestal em ciclos longos levou a um grande número de tentativas de expressar o crescimento das árvores, por meio de formulações matemáticas.

A predição de parâmetros é um procedimento amplamente empregado para predizer distribuições de variáveis aleatórias, por meio de um conjunto de variáveis exógenas relacionadas com a distribuição (TORRESROJO et al., 2000).

\footnotetext{
${ }^{1}$ Recebido em 31.08.2006 e aceito para publicação em 20.11.2006.

${ }^{2}$ Faculdade de Engenharia Florestal da Universidade Federal de Mato Grosso. E-mail:<thelsoares@ufmt.br>.

${ }^{3}$ Departamento de Engenharia Florestal da Universidade Federal de Viçosa. E-mail: <hgleite@ ufv.br>.

${ }^{4}$ Centro de Ciências Agrárias da Universidade Federal do Espírito Santo, Campus Alegre. E-mail: <gfsilva@cca.ufes.br>.
} 
A técnica de predição do crescimento e da produção de povoamentos florestais que utiliza distribuições diamétricas pode ser utilizada na modelagem de povoamentos equiiâneos e inequiiâneos. Para gerar esses modelos, funções de distribuição de probabilidade têm sido utilizadas para caracterizar as distribuições diamétricas de povoamentos florestais (MURPHY e FARRAR JR., 1988). De acordo com Bailey e Dell (1973), a função Weibull tem sido uma das mais aplicadas na área florestal devido à sua flexibilidade e capacidade para descrever diferentes tipos de distribuições.

Segundo Campos e Turnbull (1981), a função Weibull apresenta superioridade em relação às demais para os propósitos florestais devido à sua flexibilidade e à facilidade de correlacionar, de forma significativa, seus parâmetros com características importantes do povoamento.

Por essa razão, a função Weibull tem sido extensivamente utilizada em estudos de crescimento e produção florestal, com vários trabalhos publicados em literaturas nacionais e internacionais, entre eles: Smalley e Bailey (1974), Hafley e Schreuder (1977), Clutter e Belcher (1978), Clutter et al. (1983), Little (1983), Bailey et al. (1985), Magnussen (1986), Silva e Bailey (1987), Leite et al. (1990), Nogueira et al. (2001), Abreu et al. (2002) e Nogueira et al. (2005), entre outros.

A maioria dos estudos de projeção do crescimento e da produção de povoamentos florestais modela os estimadores dos parâmetros de uma função de densidade probabilística (f.d.p.) de forma explícita, ou seja, a modelagem é feita mediante modelos lineares ou nãolineares com variáveis preditoras que utilizam alguns parâmetros do povoamento, como densidade, diâmetro médio, altura dominante, índice de sítio e, ou, idade. Tal procedimento é denominado predição de parâmetros e, embora as relações lineares dos modelos possam ter fundamentos biológicos, a precisão gerada tem sido baixa em alguns casos (SMALLEY e BAILEY, 1974; CAO e BURKHART, 1984; TORRES-ROJO et al., 2000).

Tradicionalmente, os modelos de distribuição diamétrica relacionam alterações das variáveis características dos povoamentos para estimar e, ou, avaliar as alterações ocorridas ao longo do tempo. Nesse caso, as características mensuradas são empregadas como variáveis independentes nos modelos utilizados para predizer o estoque de crescimento e a produção do povoamento. De acordo com Gertner (1986, 1991), esse procedimento, usual no manejo de florestas eqüiâneas, gera erros inconvenientes que ocorrem de diferentes maneiras: erros de medição, erros de amostragem, erros de predição (em sistemas complexos da simulação, as variáveis de um modelo geralmente são preditas utilizando outros modelos, fazendo que haja a propagação dos erros de um modelo para outro).

Considerando que as informações resultantes dos modelos de crescimento são utilizadas para predizer o estoque em crescimento atual e futuro dos povoamentos para fins de planejamento e na tomada de decisões, torna-se necessário testar novos procedimentos com o intuito de minimizar os inconvenientes citados anteriormente.

Uma alternativa ao emprego da correlação de características do povoamento com parâmetros da f.d.p. foi desenvolvida por Guimarães (1994), na qual a dinâmica da estrutura de povoamentos florestais eqüiâneos se baseia na análise de medidas de posição das distribuições diamétricas. A metodologia proposta pelo autor consiste em estimar os diâmetros e alturas correspondentes aos porcentis $50 \%$ e $75 \%$ das distribuições e, com estes, recuperar e projetar as estruturas horizontais e verticais ao longo do tempo.

O modelo formulado por Guimarães (1994) é do tipo passo invariante, cuja configuração permite a obtenção de resultados compatíveis entre os volumes observados e projetados, além da utilização de dados de qualquer inventário, em qualquer tempo, como base para o estabelecimento de projeções do crescimento de um povoamento.

Embora o próprio autor tenha demonstrado a eficiência do modelo, nenhuma publicação sobre o procedimento foi encontrada nos últimos anos, com exceção de um artigo publicado por ele (GUIMARÃES, 2000), que avaliou um procedimento de passo invariante empregando a função Gompertz em dados de crescimento de frangos de corte e em parcelas permanentes de plantações de Eucalyptus grandis.

Assim, o objetivo deste estudo foi simular alterações nas estruturas horizontal e vertical de um povoamento de Eucalyptus sp., utilizando o modelo de distribuição diamétrica de passo invariante, baseado no trabalho de Guimarães (1994), bem como avaliar novamente a eficiência do modelo. 


\section{MATERIAL E MÉTODOS}

\subsection{Fonte de dados}

Este estudo foi desenvolvido com dados da primeira rotação de povoamentos de eucalipto híbrido estaca (Eucalyptus grandis x Eucalyptus urophylla), plantados no espaçamento de 3,0 x 2,0 m, localizados na região noroeste do Estado da Bahia.

Os dados são provenientes de parcelas permanentes circulares com raio de 12,25 m. Em cada parcela foram medidos os diâmetros a 1,3 m de altura do solo (dap) de todas as árvores e a altura total $(\mathrm{Ht})$ de 20 árvores por parcela. No total foram mensuradas 173 parcelas, sendo que em seis delas foram feitas duas medições; em 16, 3 medições; em 35, quatro medições; em 84, cinco medições; em 25, seis medições; e em sete, sete medições. A idade de medição variou entre 25 e 89 meses, sendo que as medições foram realizadas, anualmente, durante os anos de 1990 a 1996.

Para o ajuste da equação de volume com casca, procedeu-se à cubagem de 168 árvores-amostra, em diferentes classes de diâmetro. Diâmetros com casca foram medidos nas alturas de 0,$1 ; 0,3 ; 0,7 ; 1,0 ; 1,3$; 2,0; 4,0; e 6,0 m, e assim sucessivamente até cerca de $4 \mathrm{~cm}$ de diâmetro do fuste. $\mathrm{O}$ volume de cada árvore foi totalizado pela aplicação sucessiva da fórmula de Smalian.

A altura total e o volume das árvores das parcelas foram obtidos, empregando-se equações referentes aos seguintes modelos:

$$
\begin{aligned}
& \ln H t=\theta_{0}+\theta_{1} d a p^{-1}+\theta_{2} \ln (H d)+\ln \left(\varepsilon_{i}\right) \\
& V=\theta_{0} d a p^{\theta_{1}} H t^{\theta_{2}}\left[e^{\theta_{3}(T x / d a p)}\right]\left[1-\left(d \times d a p^{-1}\right)^{1+\theta_{4} d}\right]+\varepsilon_{i}
\end{aligned}
$$

em que:

$$
H t=\text { altura total }(\mathrm{m}) \text {; }
$$

dap $=$ diâmetro com casca medido a 1,3 m de altura do solo $(\mathrm{cm})$;

$$
\begin{aligned}
& H d=\text { altura dominante }(\mathrm{m}) ; \\
& V=\text { volume com casca }\left(\mathrm{m}^{3}\right) ; \\
& d=\text { diâmetro comercial }(\mathrm{cm}) ; \\
& l n=\text { logaritmo neperiano; } \\
& e=\text { base dos logaritmos neperianos; } \\
& T x=\text { variável binária }(0 \text { para volume ou diâmetro } \\
& d \text { com casca e } 1 \text { para volume ou diâmetro } d \text { sem casca); }
\end{aligned}
$$

$$
\begin{aligned}
& \theta_{\mathrm{i}}=\text { parâmetros dos modelos }(i=0,1, \ldots, 4) ; \mathrm{e} \\
& \varepsilon_{i}=\text { erro aleatório, } \mathrm{e} \sim \mathrm{N}\left(0, \sigma^{2}\right) .
\end{aligned}
$$

\subsection{Distribuições diamétricas}

O procedimento denominado passo invariante (step invariant) baseia-se no princípio de que predições sucessivas, efetuadas da idade $I_{1}$ para $I_{2}$ e da idade $I_{2}$ para $I_{3}$, devem, matematicamente, apresentar as mesmas predições, obtidas por um único passo entre as idades $I_{1}$ e $I_{3}$ (SOMERS e FARRAR JR., 1991).

Segundo Guimarães (1994), nesse procedimento as medidas de posição, efetuadas na distribuição em determinada idade, constituem a base da estimativa das magnitudes dessas mesmas medidas em outras idades.

Para o ajuste de um modelo de passo invariante, os dados relativos ao número de árvores por classe de diâmetro foram ordenados de forma acumulativa. Dessa forma, a classe de maior diâmetro inclui o número total de árvores contidas na parcela.

A distribuição diamétrica foi estimada pela função Weibull com apenas dois parâmetros, cuja f.d.p. é dada por:

$$
f(x)=\frac{\gamma}{\beta} \cdot\left(\frac{x}{\beta}\right)^{\gamma-1} \exp \left[-\left(\frac{x}{\beta}\right)^{\gamma}\right]
$$

em que:

$\beta=$ parâmetro de escala;

$\gamma=$ parâmetro de forma; e

$x=$ variável aleatória, no caso centro de classe de dap, sendo $\mathrm{x} \geq 0, \beta>0, \gamma>0$.

Os dados foram agrupados em classes com intervalos regulares de $1 \mathrm{~cm}$ de amplitude, e a função foi ajustada aos dados de distribuição diamétrica observada de cada parcela em todas as medições. Para a estimação dos parâmetros da f.d.p., foi empregado o método da máxima verossimilhança, sendo utilizado o algoritmo proposto por Gove e Fairweather (1989), o qual foi implementado em uma planilha do Excel.

A partir das estimativas dos parâmetros e da função de distribuição Weibull, obtiveram-se as freqüências estimadas do número de árvores por classe diamétrica. A proporção da distribuição localizada entre dois valores

R. Árvore, Viçosa-MG, v.31, n.2, p.275-283, 2007 
específicos de $x$, como $x_{\mathrm{i}}$ e $x_{\mathrm{s}}\left(x_{\mathrm{i}} \leq x_{\mathrm{s}}\right)$, é definida como:

$$
\mathrm{p}\left(\mathrm{x}_{\mathrm{i}}, \mathrm{x}_{\mathrm{s}}\right)=\exp \left[-\left(\frac{x_{i}}{\beta}\right)^{\gamma}\right]-\exp \left[-\left(\frac{x_{s}}{\beta}\right)^{\gamma}\right]
$$

em que:

$x_{\mathrm{i}}=$ limite inferior da classe de diâmetro;

$x_{\mathrm{s}}=$ limite superior da classe de diâmetro; e

$\beta$ e $\gamma=$ conforme definido anteriormente.

Após obter as estimativas dos parâmetros da função Weibull, efetuou-se a linearização do modelo [3], conforme procedimento adotado por Krug et al. (1984) e Guimarães (1994):

$$
\ln \ln [1 /(1-Y / A)]=\beta+Y \ln (X)
$$

A obtenção de qualquer percentil da distribuição, de acordo com McTague e Bailey (1987), passa a ser definida por:

$$
\ln \ln [1 /(1-\text { percentil })]=\beta+g \ln (X)
$$

No presente caso, uma vez que apenas dois parâmetros caracterizam a função Weibull ( $\beta$ e $\gamma$ ), com base na metodologia proposta por Guimarães (1994), selecionaram-se os percentis correspondentes a 50 e $75\left(\mathrm{P}_{50}\right.$ e $\left.\mathrm{P}_{75}\right)$, cujas estimativas são dadas por:

$$
\begin{aligned}
& \mathrm{P}_{50}=\ln \ln [1 /(1-0,5)]=\hat{\beta}+\hat{\gamma} \ln (\mathrm{X}) \\
& \mathrm{P}_{50}=\exp [(-0,3665-\ln \hat{\beta}) / \hat{\gamma}] \\
& \mathrm{P}_{75}=\ln \ln [1 /(1-0,75)]=\hat{\beta}+\hat{\gamma} \ln (\mathrm{X}) \\
& \mathrm{P}_{75}=\exp [(0,3266-\ln \hat{\beta}) / \hat{\gamma}] \\
& \text { em que: }
\end{aligned}
$$

$P_{50}=$ percentil correspondente a $50 \%$ da distribuição;

$P_{75}=$ percentil correspondente a $75 \%$ da distribuição;

ln = logaritmo neperiano;

$\hat{\beta}=$ parâmetro de expansão da distribuição Weibull; e

$\hat{\gamma}=$ parâmetro de forma da distribuição Weibull.

A partir dessas medidas de posição, torna-se possível estimar os percentis em idades futuras. A obtenção das posições futuras baseou-se no princípio de que medidas de posição, efetuadas na distribuição em determinada idade, constituam a base de estimativa das magnitudes dessas mesmas medidas também em outras idades, efetuando projeções da distribuição diamétrica e da relação hipsométrica.

As projeções da distribuição diamétrica foram baseadas em um modelo de percentis futuros, uma vez que variações nos percentis determinam curvas de crescimento similares às do crescimento diamétrico. $\mathrm{O}$ seguinte modelo foi ajustado para estimar $\mathrm{Pf}_{50}$ e $\mathrm{Pf}_{75}$ :

$$
P_{f}=\left(1-e^{-\beta_{0} I_{f}{ }^{\beta_{1}} P_{a}^{\beta_{2}}}\right) P_{a} /\left(1-e^{-\beta_{0} I_{a}^{\beta_{1}} P_{a}^{\beta_{2}}}\right)+\varepsilon_{i}
$$

em que:

$$
\begin{aligned}
& P_{f}=\text { percentil na idade futura; } \\
& P_{a}=\text { percentil na idade atual; } \\
& I_{a}=\text { idade atual de medição; e } \\
& I_{f}=\text { idade futura. }
\end{aligned}
$$

De posse dos estimadores dos parâmetros das equações, obtiveram-se, mediante regressão linear simples, as estimativas dos parâmetros da função Weibull, e, então, efetuou-se a reconstituição destas.

Os parâmetros da função Weibull foram estimados por:

$$
\hat{\beta}=\exp \left(\hat{\beta}_{0}\right) \therefore \hat{\gamma}=\hat{\beta}_{1}
$$

em que:

$$
\begin{aligned}
& \hat{\beta}_{0}=\bar{Y}-\hat{\beta}_{1} \bar{X} ; \mathrm{e} \\
& \hat{\beta}_{1}=\frac{\sum X Y-\frac{\sum X \sum Y}{n}}{\sum X^{2}-\frac{\left(\sum X\right)^{2}}{n}}
\end{aligned}
$$

sendo:

$X=$ valores estimados para os percentis futuros, sendo representados por $X_{1}$ e $X_{2}$;

$$
\begin{aligned}
& X_{1}=\ln \left(P_{f} 50\right) ; \\
& X_{2}=\ln \left(P_{f} 75\right) ; \\
& Y=\text { valores de } \mathrm{Y}_{1} \mathrm{e} \mathrm{Y}_{2} ; \\
& Y_{1}=\ln \ln (1 / 1-0,50)=-0,3665 ; \mathrm{e} \\
& Y_{2}=\ln \ln (1 / 1-0,75)=0,3266 .
\end{aligned}
$$


As freqüências estimadas foram obtidas após recuperar os parâmetros da distribuição Weibull. Verificou-se que a recuperação da distribuição foi baseada, apenas, nos percentis na idade de medição do povoamento $\left(I_{a}\right)$ e na idade futura de projeção $\left(I_{f}\right)$.

A projeção das relações hipsométricas foi realizada por meio de estimativas das alturas referentes aos percentis 50 e $75\left(H R_{50}\right.$ e $\left.H R_{75}\right)$, sendo ajustado o modelo:

$$
H R_{f}=H R_{a}\left[e^{-\beta_{0}\left(I_{a}-1-I_{f}-1\right) \cdot H R_{a} \beta_{1}}\right]^{-1}+\varepsilon_{i}
$$
em que:

$H R_{f}=$ altura relativa ao percentil, considerado $\left(H R_{50}\right.$ ou $H R_{75}$ ) na idade futura;

$H R_{a}=$ altura na idade atual; e

$I_{a}, I_{f}, \varepsilon_{i}=$ conforme definidos anteriormente.

Enquanto as projeções dos percentis $\left(P_{50}\right.$ e $\left.P_{75}\right)$ fornecem estimativas dos diâmetros correspondentes a essas posições da distribuição na idade futura considerada, as projeções das alturas relativas $\left(H R_{50}\right.$ e $H R_{75}$ ) fornecem estimativas das alturas correspondentes aos percentis $P_{50}$ e $P_{75}$, e, portanto, aos respectivos diâmetros na idade referenciada.

\subsection{Validação do modelo}

De posse dos parâmetros estimados, curvas teóricas da distribuição foram obtidas para diferentes povoamentos, ao substituí-los na f.d.p. A distribuição diamétrica foi projetada a partir de uma distribuição em uma idade atual para uma idade futura conhecida, partindo-se sempre de uma distribuição inicial observada.

O teste de compatibilidade consistiu em analisar se a distribuição diamétrica projetada do início para o final de um período de tempo é compatível com a distribuição diamétrica projetada ano a ano. Para tanto, empregou-se o teste Qui-quadrado $\left(\chi^{2}\right)$, em nível de $1 \%$ de probabilidade, para testar as seguintes hipóteses:

$H_{0}=$ A distribuição do número de árvores por classe de diâmetro no final do período projetado da idade inicial $I_{1}$ para $I_{2}, I_{2}$ para $I_{3}, \ldots, I_{n-1}$ para $I_{n}$ é igual à distribuição do número de árvores por classe de diâmetro no final do período projetado de $I_{l}$ para $I_{n}$; e

$$
H_{a}=\text { rejeita-se } \mathrm{H}_{0} \text {. }
$$

em que:

$$
\begin{aligned}
& I_{I}=\text { idade atual (meses); } \mathrm{e} \\
& I_{i}=\text { idade futura (meses), }(i=2, \ldots, \mathrm{n}) .
\end{aligned}
$$

Estimativas de volume por hectare foram obtidas a partir da distribuição diamétrica observada e das projeções da distribuição das estruturas horizontal e vertical. A equação de múltiplos volumes foi, então, aplicada para obter as estimativas da produção total e por classe de diâmetro para todas as parcelas e em todas as medições. Para a comparação dos volumes prognosticados com os volumes observados por classe diamétrica, utilizou-se o teste L\&O proposto por Leite e Oliveira (2002), cujo procedimento resulta da combinação do teste de $\mathrm{F}\left(\mathrm{H}_{0}\right)$ Graybill, do teste t para erro médio $(\mathrm{t} \bar{e})$ e da análise de correlação linear $\left(r_{y j y l}\right)$ entre os valores observados $\left(\mathrm{Y}_{\mathrm{j}}\right)$ e os valores estimados $\left(\mathrm{Y}_{1}\right)$. O teste compara o valor observado como variável dependente e o valor estimado como variável independente. Assim, para situações em que $\mathrm{F}\left(\mathrm{H}_{0}\right)$ e te forem não-significativos (ns) e $r_{y j y l} \geq 1-|\bar{e}|$, concluise que os valores estimados pela regressão não diferem dos observados.

\section{RESULTADOS E DISCUSSÕES}

\subsection{Modelos hipsométrico e volumétrico}

Os modelos hipsométrico e volumétrico utilizados para estimar volumes por árvore resultaram em estimativas precisas e livres de tendências (Figura 1), sendo:

$$
\begin{aligned}
& \ln H t=0,81574+0,48837 \text { dap }^{-1}-2,53925 \ln (H d) \\
& \mathbf{r}_{\hat{\mathrm{y} y}}=0,977 \\
& V=0,00008 \cdot d a p^{1,53553} \cdot H t^{1,44819} \cdot\left[e^{-2,25669(T x / d a p)}\right] . \\
& \cdot\left[1-\left(d \cdot d a p^{-1}\right)^{1-0,00077 \cdot d}\right] \\
& \mathbf{r}_{\hat{\mathrm{y}} \mathrm{y}}=0,999
\end{aligned}
$$

\subsection{Modelagem do crescimento}

\subsubsection{Projeção das distribuições diamétricas e hipsométricas}

Após ajustar a f.d.p. Weibull à distribuição diamétrica observada e calcular os percentis $\left(P_{50} \mathrm{e}\right.$ $\left.P_{75}\right)$ da distribuição, estimaram-se os percentis em idades futuras empregando as equações ajustadas para projeção das alterações dos percentis ao longo do tempo:

$$
\begin{aligned}
& \mathrm{P}_{\mathrm{f}_{50}}=\left(1-\mathrm{e}^{-30,36807 \mathrm{I}} \mathrm{f}^{-0,72565} \mathrm{~Pa}_{50}{ }^{-1,26441}\right) \text {. } \\
& . \mathrm{P}_{50} /\left(1-\mathrm{e}^{-30,36807 \mathrm{I}_{\mathrm{a}}}{ }^{-0,72565} \mathrm{~Pa}_{50}{ }^{-1,26441}\right) \\
& \mathbf{r}_{\hat{y} y}=0,908
\end{aligned}
$$




$$
\begin{aligned}
& \mathrm{P}_{\mathrm{f} 75}=\left(1-\mathrm{e}^{\left.-4,11011 \mathrm{I}_{\mathrm{f}}{ }^{-0,25093} \mathrm{~Pa}_{50}{ }^{-0,23489}\right)}\right) \\
& . \mathrm{P}_{\mathrm{a}_{50}} /\left(1-\mathrm{e}^{\left.4,11011 \mathrm{I}_{\mathrm{a}}{ }^{-0,25093} \mathrm{~Pa}_{50}{ }^{-0,23489}\right)}\right. \\
& \mathrm{r}_{\hat{\mathrm{y} y}}=0,912
\end{aligned}
$$

Segundo Guimarães (1994), a aplicação dessas equações, por incluir o próprio percentil como variável independente, garante que diferentes tendências de crescimento das variáveis $P_{50}$ e $P_{75}$ estejam relacionadas com a magnitude destas, permitindo, portanto, que a estrutura do povoamento constitua importante indicativo da capacidade produtiva do local. A relação entre os percentis estimados e observados e a distribuição de resíduos é apresentada na Figura 2.

Uma peculiaridade importante desse modelo ajustado para a projeção dos percentis reside no fato de efetuarem a projeção a partir da idade em que as medições foram efetuadas no campo. Como se trata de um modelo totalmente compatível, as projeções podem ser realizadas, objetivando definir produções em épocas passadas. Portanto, a partir de uma única medição, torna-se possível estabelecer a provável tendência de crescimento da população, o que caracteriza a propriedade invariante do modelo (GUIMARÃES, 1994).

Nesse caso, a projeção da distribuição foi realizada com base apenas nos percentis na idade de medição e na idade futura de projeção. As projeções realizadas foram comparadas com os valores observados por meio do teste Qui-quadrado $\left(\chi^{2}\right)$, sendo que $94,9 \%$ das projeções efetuadas apresentaram distribuições estatisticamente semelhantes à observada.

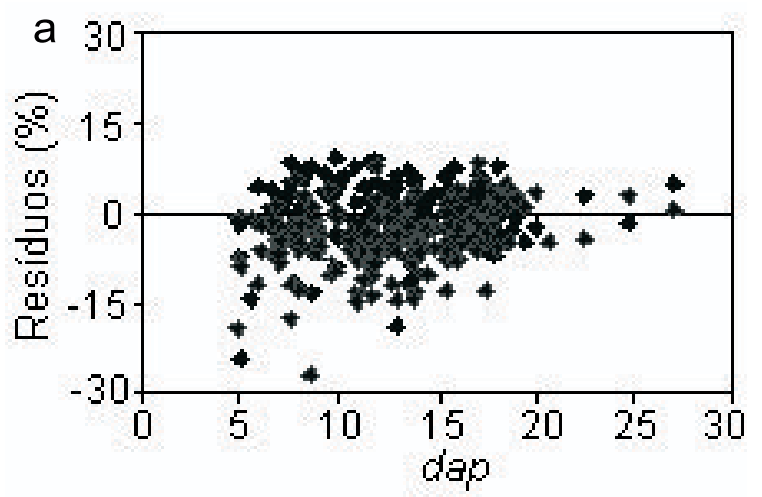

Para projetar as relações hipsométricas ao longo do tempo, foi necessário estimar as alturas correspondentes aos diâmetros relativos aos percentis de 50 e 75 e, em seguida, projetar as alturas relativas (HR), empregando-se as equações de projeções futuras das alturas, cujos ajustes foram:

$$
\begin{aligned}
& \left.H R_{f 50}=H R_{a} \cdot\left[e^{-312,8691\left(I_{a}^{-1}-I_{f}^{-1}\right) \cdot H R_{a}}\right]^{-0,5776}\right]^{-1} \\
& \mathbf{r}_{\hat{\mathrm{yy}}}=0,889 \\
& H R_{f 75}=H R_{a} \cdot\left[e^{-299,3866\left(I_{a}{ }^{-1}-I_{f}^{-1}\right) \cdot H R_{a}}{ }^{-0,5840}\right]^{-1} \\
& \mathbf{r}_{\hat{\mathrm{y}} \mathrm{y}}=0,884
\end{aligned}
$$

Na Figura 3 é apresentada a relação entre as alturas relativas observadas e estimadas.

\subsection{Validação das projeções}

Após obter as distribuições diamétricas e relações hipsométricas em todas as idades, aplicou-se a equação [9] para estimar a produção volumétrica em diferentes idades.

Na Figura 4 é apresentado um exemplo dos resultados de aplicação da equação volumétrica e do modelo de distribuição diamétrica para a estimativa do volume total com casca (VTcc) e do volume comercial com casca (VCcc) - nesse caso considerado até o diâmetro aproveitável de $6 \mathrm{~cm}$-e para a energia com casca (VEcc) para algumas parcelas do povoamento em diferentes idades.

A comparação das projeções volumétricas foi feita pelo teste $\mathrm{L} \& \mathrm{O}$, cujo resultado indicou identidade entre as distribuições com $\mathrm{F}<1^{\text {ns; }} ; t_{\bar{e}}=1,498^{\text {ns. }} ;$ e $r_{y j y 1} \geq 1-|\bar{e}|$. Uma ilustração desse resultado é apresentada na Figura 5.

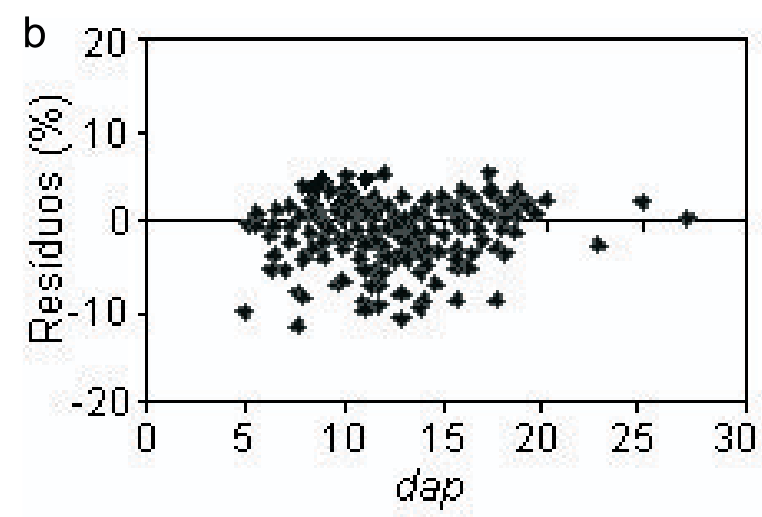

Figura 1 - Distribuição de resíduos porcentuais em relação ao dap: a) equação hipsométrica e b) equação volumétrica. Figure 1-Residue distribution in percentage: a) hypsometric equation; $b$ ) volumetric equation. 

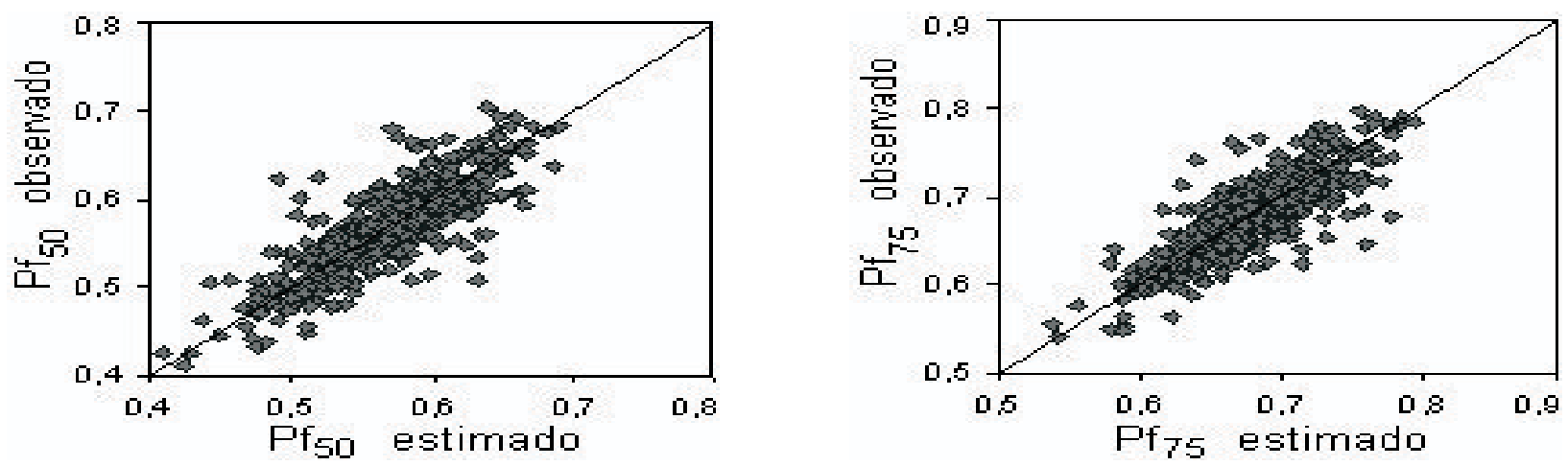

Figura 2 - Relação entre os percentis estimados e observados. Figure 2-Estimated and observed percentile relationship.
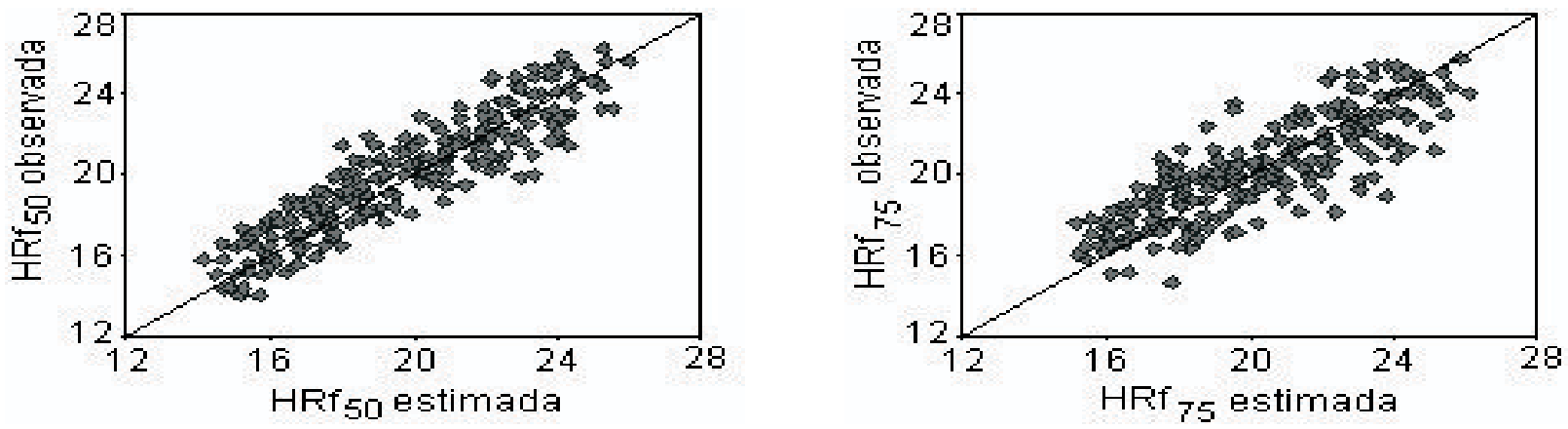

Figura 3 - Relação entre as alturas relativas estimadas e observadas.

Figure 3-Estimated and observed relative height relationship.
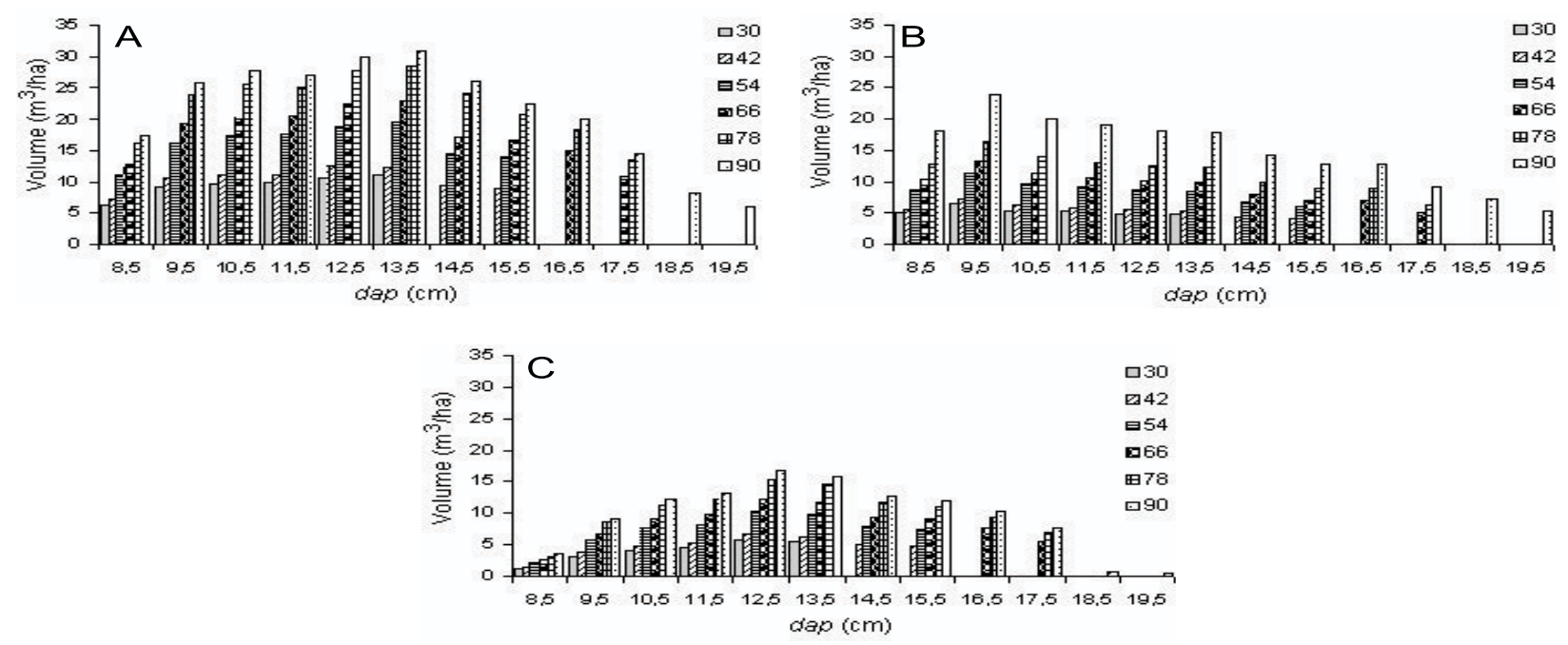

Figura 4 - Projeção da produção volumétrica nas idades de 30, 42, 54, 66, 78 e 90 meses: a) volume total com casca (VTcc), b) volume comercial com casca (VCcc); e c) volume para energia com casca (VEcc).

Figure 4-Volumetric production projection at the ages 30, 42, 54, 66, 78 and 90 months. Where: a) Total volume with bark (VTcc); b) Commercial volume with bark (VCcc); c) Volume for energy with bark (VEcc). 
a

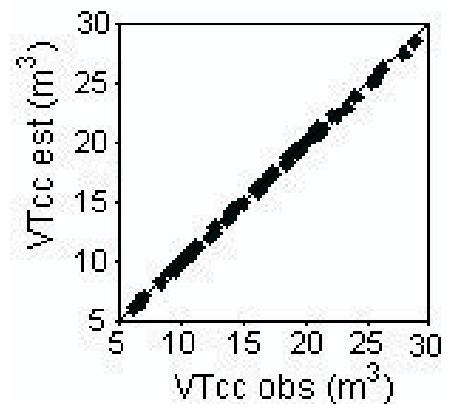

b

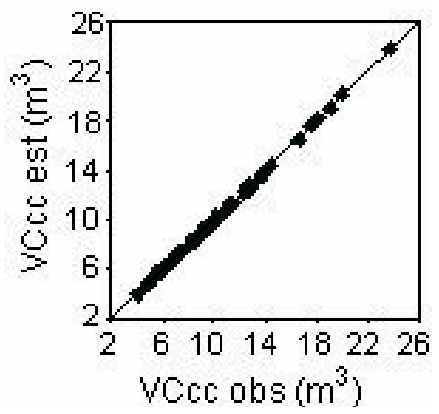

C

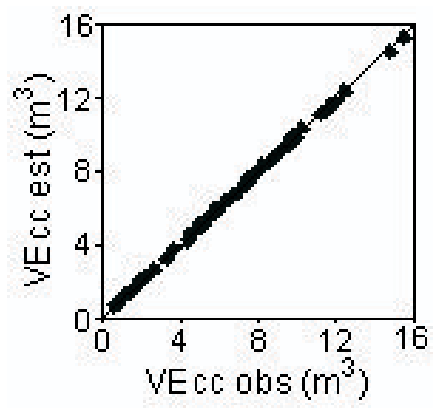

Figura 5 - Relação entre volumes observados versus volumes estimados: VTcc = volume total com casca, VCcc $=$ volume comercial com casca e VEcc = volume para energia com casca.

Figure 5-Observed and estimated volume relationship: Where: VTcc = total volume with bark; VCcc = commercial volume with bark; VEcc = volume for energy with bark.

Por efetuar projeções a partir da idade de medição, a precisão com que os parâmetros da função Weibull são recuperados é dependente da idade inicial utilizada e do período da projeção para o qual a projeção for efetuada. Essa característica representa uma importante vantagem da utilização do modelo aplicado em relação aos usualmente empregados para a projeção do crescimento e da produção.

Guimarães (1994), ao descrever a metodologia do modelo de distribuição diamétrica de passo invariante aplicado nesse estudo e realizar testes de aplicação e validação do modelo em plantações de eucaliptos instaladas na região do Vale do Rio Doce, Minas Gerais, observou que as tendências de crescimento, definidas pelo modelo para as estruturas horizontais e verticais dos povoamentos, estão em conformidade com aquelas esperadas de crescimento sob os preceitos biológicos.

Outra vantagem da metodologia aplicada refere-se à simplicidade de aplicação do método e também à minimização do número de variáveis envolvidas na recuperação da f.d.p.

\section{CONCLUSÕES E RECOMEDAÇÕES}

Os resultados permitiram concluir que a metodologia de passo invariante empregada é consistente por apresentar tendência semelhante detectada entre os volumes projetados e os observados. Não houve tendenciosidade em nenhuma das etapas da modelagem da predição das estruturas do povoamento. Devido à simplicidade de ajuste do modelo, o qual é baseado apenas em percentis da distribuição diamétrica, recomenda-se o seu emprego no manejo de povoamentos de eucalipto não desbastados.

R. Árvore, Viçosa-MG, v.31, n.2, p.275-283, 2007

\section{AGRADECIMENTOS}

Ao CNPq pela concessão da bolsa de estudos da primeira autora.

\section{REFERÊNCIAS}

ABREU, E.C.R. et al. Modelagem para prognose precoce do volume por classe diamétrica para Eucalyptus grandis. Scientia Forestalis, n.61, p.86-102, 2002.

\section{ASSMANN, B. The principles of forest} yield study. Oxford: Pergaman Press, 1970. 506p.

BAILEY, R.L.; DELL, T.R. Quantifying diameter distributions with the Weibull function. Forest Science, v.19, n.2, p.97-104, 1973.

BAILEY, R. L. et al. A compatible model relating slash pine plantation survival to density, age, site index and type and intensity of thinning. Forest Science, v.31, n.1, p.181-189, 1985.

CAMPOS, J. C. C.; TURNBULL, K. J. Um sistema para estimar produção por classe de diâmetro e sua aplicação na interpretação do efeito de desbaste. Revista Árvore, v.5, n.1, p.1-16, 1981 .

CAO, Q.V.; BURKHART, H.E. A segmented distribution approach for modeling diameter frequency data. Forest Science, v.30, n.1, p.129-137, 1984. 
CLUTTER, J.L.; BELCHER, D.M. Yield of siteprepared slash pine plantations in the lower coastal, plain of Georgia and Florida. In: GROWTH MODELS FOR LONG-TERM FORECASTING OF TIMBER YIELDS, 1978, Blacksburg. Proceedings... Blacksburg: IUFRO, School of Forestry and Wildlife Resources, State Unversity, 1978. p.53-70.

GERTNER, G.Z. Postcalibration sensitivity procedure for regressor variable error. Canadian Journal of Forest Research, v.16, p.1120-1123, 1986.

GERTNER, G.Z. Prediction bias and response surface curvature. Forest Science, v.37, n.3, p.755-765, 1991 .

GUIMARÃES, D.P. Desenvolvimento de um modelo de distribuição diamétrica de passo invariante para prognose e projeção da estrutura de povoamentos de eucalipto. 1994. 160f. Tese (Doutorado em Ciência Florestal) - Universidade Federal de Viçosa, Viçosa, MG, 1994.

GOVE, J.H.; FAIRWEATHER, S.E. Maximumlikelihood estimation of Weibull function parameters using a general interactive optimizer and grouped data. Forest Ecology and Management, v.28, n.1, p.61-69, 1989.

HAFLEY, W.L.; SCHREUDER, H.T. Stastistical distributions for fitting diameter and height data in even-aged stands. Canadian Journal Forest Research, v.7, p.481-487, 1977.

KRUG, A.G.; NORDHEIN, E.V.; GIESE, R.L. Determining initial values for parameters of a Weibull model: a case study. Forest Science, v.30, n.3, p.573-581, 1984.

LEITE, H. G. et al. Um modelo para estimar a produção de madeira para celulose e energia derivado da função Weibull. Revista Árvore, v.14, n.2, p.102-118, 1990.

LEITE, H.G.; OLIVEIRA, F.H.T. Statistical procedure to test the identity of analytical methods.

Communications in Soil Science Plant Analysis, v.33, n.7/8, p.1105-1118, 2002.
LITTLE, S.N. Weibull diameter distributions for mixed stands of western conifers. Canadian Journal of Forest Research, v.13, n.1, p.85-88, 1983.

McTAGUE, J.P.; BAILEY, R.L. Compatible basal area and diameter distribution models for thinned loblolly pine plantations in Santa Catarina, Brazil. Forest Science, v.33, n.1, p.43-51, 1987.

MAGNUSSEN, S. Diameter distribution in picea abies described by the Weibull model. Scandinavian Journal Forest Research, n.1, p.493-502, 1986.

MURPHY, P.A.; FARRAR JR., R.M. A framework for stand structure projection of uneven-aged loblolly-shortleaf pine stands. Forest Science, v.34, n.2, p.321-332, 1988.

NOGUEIRA, G.S. et al. determinação da idade técnica de desbaste em plantações de eucalipto utilizando o método dos ingressos percentuais. Scientia Forestalis, n.59, p.51-59, 2001.

NOGUEIRA, G.S. et al. Modelo de distribuição diamétrica para povoamentos de Eucalyptus sp. submetidos a desbaste. Revista Árvore, v.29, n.4, p.579-589, 2005.

\section{SMALLEY, G.W.; BAILEY, R.L. Yield tables and stand structure for loblolly pine plantations in Tennessee, Alabama and Georgia highlands. Washington: USDA, 1974. 81p. (Forest Service Research Paper SO, 96)}

SILVA, J.A.A.; BAILEY, R.A. Predicting diameter distribution in fertilized slash pine plantations with the Weibull distribution. Acta Forestalia Brasiliensis, v.2, n.1, p.47-62, 1987.

SOMERS, G.L.; FARRAR JR., R.M.

Biomathematical growth equations for natural longleaf pine stands. Forest Science, v.37, n.1, p.227-244, 1991.

TORRES-ROJO, J.M.; MAGAÑA-TORRES, O.S.; ACOSTA-MIRELES, M. Metodología para mejorar la predicción de parámetros de distribuciones. Agrociencia, v.34, n.5, p.627-637, 2000. 